\title{
Special issue on multi-agent systems
}

\author{
Nils Bulling 1
}

Published online: 24 May 2016

(C) Springer International Publishing Switzerland 2016

\section{Introduction}

A multi-agent system is a system composed of a number of intelligent agents that can interact with each other and an environment. The research community concerned with multi-agent systems is very interdisciplinary, including researchers from disciplines like mathematics, machine learning, game theory, logics, sociology, philosophy, software engineering and many more. Similarly diverse is the set of possible applications across a huge number of domains like automotive, aerospace, manufacturing, traffic, defence, health care, and e-commerce. This interdisciplinarity is very much in the spirit of the European Conference on Multi-Agent Systems ${ }^{1}$ (EUMAS). The aim of EUMAS is to encourage and support activity in the research and development of multi-agent systems, and to provide a forum for researchers from academia as well as from industry to meet, to present their work, and to discuss ideas in a friendly and professional environment. The first edition of EUMAS was held in Oxford in 2003, followed by editions in Barcelona 2004, Brussels 2005, Lisbon 2006, Hammamet 2007, Bath 2008, Agia Napa 2009, Paris 2010, Maastricht 2011, Dublin 2012, and Toulouse 2013. Finally, EUMAS 2014 was held in Prague where it was run, for the first time, as a conference with formal proceedings (LNCS/LNAI, Springer).

The present special issue consists of a selection of the best papers submitted to EUMAS 2014. Authors of selected papers of the conference were invited to submit revised and extended versions of their conference paper to this special issue. All submissions had to pass a fresh, high quality reviewing round along the standards of the journal. The selection process resulted in four papers covering different aspects of multi-agent systems, ranging from theoretical work on the synthesis of strategies in a game theoretical context, over a

${ }^{1}$ Until 2014 EUMAS was known as European Workshop on Multi-Agent Systems.

Nils Bulling

n.bulling@tudelft.nl

1 Department of Intelligent Systems, TU Delft, Mekelweg 4, 2628 CD Delft, Netherlands 
new methodology to design agent-based simulations, to questions about crowdsourcing and the coordination of emergency medical services.

Kupferman, Perelli and Vardi study Synthesis with Rational Environments. The authors expand on the rational synthesis problem which is defined as follows: given the objectives of agents constituting the environment and of the system, compute a strategy profile which satisfies the system's objective and which is in equilibrium for the agents constituting the environment. The authors introduce strong rational synthesis where the environment agents may not be cooperative and can follow any strategy in equilibrium, not just the synthesised one. Furthermore, the authors use a quantitative version of the linear-time temporal logic LTL to specify objectives, and allow coalitions of agents that constitute the system or the environment. Finally, the authors show how the different synthesis problems can be reduced to the model checking problem of fragments of Strategy Logic.

In the second contribution, Klügl argues that the "identification of relevant interactions and their particular design in a simulation model is one of the central challenges in developing agent-based simulation models" and promotes Using the Affordance Concept for Model Design in Agent-Based Simulation. The affordance concept is rooted in ecological philosophy and essentially captures - in multi-agent terms - the relation between an object and an agent indicating the possible actions the agent can perform with/on this object. As an example, Klügl mentions a "bench that affords sitting on it". In the present article, the author puts forward a methodology for using the affordance concept and considers how the concept can be integrated in the decision making process of agents.

In the third paper, Carvalho, Dimitrov and Larson investigate How many Crowdsourced Workers Should a Requester Hire? The authors use the crowdsourcing platform Amazon Mechanical Turk to empirically investigate this question. The study shows that the quality of the solution increases with an increasing number of workers, and in particular, that the optimal number of crowdsourced workers, in the sense that the improvement obtained by further workers is neglectable, is around 10 to 11 .

Finally, the Distributed Coordination of Emergency Medical Service for Angioplasty patients is investigated by Lujak, Billhardt and Ossowski. Their work shows how multiagent approaches can be used to develop solutions for problems in the real world. The authors propose a decision-support system for the coordination of emergency medical services aiming at the reduction of patient delay times, with a focus on patients awaiting angioplasty. In particular, the authors put forward a multi-agent architecture using an auction-based approach for coordination, and evaluate their approach by a simulation.

I believe that these four articles form a very interesting account to research in multi-agent systems and hope that you will enjoy reading.

Finally, I would like to thank all authors contributing to this special issue, the reviewers and the members of the programme committee of EUMAS 2014. Last but not least, I thank the Editor-in-Chief, Martin Golumbic, and the associated editor Jürgen Dix for supporting this volume.

May 2016

Nils Bulling 